\title{
Health risk appraisal for older people 5: self-efficacy in patient-doctor interactions
}

\author{
Mareeni Raymond ${ }^{1}$, Steve Iliffe ${ }^{1}$, Kalpa Kharicha $^{1}$, Danielle Harari ${ }^{2}$, Cameron Swift ${ }^{3}$, Gerhard Gillmann ${ }^{4}$ \\ and Andreas E. Stuck ${ }^{5}$ \\ ${ }^{1}$ Department of Primary Care and Population Sciences, University College London, Hampstead Campus, London, UK \\ ${ }^{2}$ Department of Ageing and Health, St. Thomas' Hospital, London, UK \\ ${ }^{3}$ Kings College London, Clinical Age Research Unit, Kings College Hospital, London, UK \\ ${ }^{4}$ Department of Social and Preventive Medicine, University of Bern, Bern, Switzerland \\ ${ }^{5}$ University Department of Geriatrics, Spital Bern-Ziegler and Inselspital, Morillonstr, Bern, Switzerland
}

\begin{abstract}
Objective: Enhancing self-efficacy is central to programmes promoting self-care and self-management. However, little is known about older people's self-efficacy in doctorpatient interactions. This paper investigates lifestyle, medical and demographic factors associated with self-efficacy in doctor-patient interactions in older people in general practice. Methods: A cross-sectional analysis of data from a randomised controlled trial of older people was conducted in a health risk appraisal study in London. Self-efficacy was measured using the Perceived Efficacy in Patient-Physician Interactions Questionnaire. Results: Older people with higher self-efficacy were significantly more likely to report having had recent preventive care measures such as recent blood pressure measurement and influenza immunisation. Women were less likely to have higher self-efficacy than men. Older people were significantly less likely to have high self-efficacy if they reported having poor memory, low mood, limited activities due to fear of falling, basic education, difficulties with at least one activity of daily living, reduced physical activity, living alone, or risk of social isolation. Conclusion: $A$ third of people had low self-efficacy in doctor-patient interactions. They appear to be a vulnerable group. Low self-efficacy in interactions with doctors may be a symptom or a characteristic of older people who experience social isolation and depression. Policies that depend on enhancing self-care and self-management need to consider the large number of older people with low self-efficacy in using medical services, and understanding characteristics in older people associated with lower confidence in doctorpatient interactions may be useful in clinical practice and research.
\end{abstract}

Keywords: older people; preventive care; primary care; self-efficacy

Received 3 March 2011; accepted 9 June 2011; first published online 21 July 2011

\section{Introduction}

Self-efficacy theory was described by Bandura in 1977 (Bandura, 1977). It proposes that a person's belief or self-confidence in the ability to undertake

Correspondence to: Dr Mareeni J. Raymond, Department of Primary Care and Population Sciences, University College London, Hampstead Campus, Rowland Hill Street, London NW3 2PF, UK. Email: mareeni_R@yahoo.com a task is a precursor for initiating behavioural change. Thus, it is a central concept in the self-care and self-management of a person's health.

Self-efficacy appears to be important (Lorig et al., 1996). First, the strength of belief that a particular result can be achieved predicts motivation and behaviour. The higher a person's selfefficacy, the more likely they are to put effort into a task, and recover from setbacks in the process. Second, self-efficacy can be enhanced by acquiring 
skills (performance mastery), learning from others (modelling), re-interpretation of symptoms, as well as through social persuasion. Self-efficacy reflects a person's optimistic self-belief about being able to change and adopt a healthy lifestyle (Strecher et al., 1986), and is strengthened when patients succeed in solving patient-identified problems (Bodenheimer et al., 2002). Third, enhanced self-efficacy leads to improved behaviour, motivation, thinking patterns and emotional well-being. Belief in one's efficacy to exercise control is thought to be a common pathway through which psychosocial influences affect health functioning (Bandura, 2004). Finally, self-efficacy, unlike dimensions of personality, seems to be specific to problems or situations (Gerin et al., 1995). Enhancing self-efficacy in one domain does not increase it in another, and different measures are needed to assess self-efficacy for each health-related behaviour being studied.

Since Bandura's concept of self-efficacy was introduced, many scales have been developed to measure self-efficacy for particular aspects of health care. Two such examples are the Medication Adherence Self-Efficacy Scale (Ogedegbe et al., 2003) and the Self-Efficacy for Exercise Scale (Resnick and Jenkins, 2000). In this paper, we focus on self-efficacy in doctor-patient interactions, using the Perceived Efficacy in Patient-Physician Interactions Questionnaire (PEPPI) scale.

A database search of titles of published studies in peer-reviewed journals such as AMED, BNI, EMBASE, HMIC, MEDLINE, PsycINFO and CINAHL, using self-efficacy, communication, confidence, interaction and older, elderly, doctor, health care or professional as keywords, showed only 13 articles, none of which focussed on older people's self-efficacy or confidence in interactions with doctors.

To the best of our knowledge, this is the first study to explore factors associated with self-efficacy in doctor-patient interactions in older people.

However, there are many studies from different continents that use self-efficacy as a measurable variable, and which show that interventions can alter self-efficacy. Improvements in self-efficacy in a cohort of people with rheumatoid arthritis receiving educational programmes as part of routine clinical care were measured in one UK study (Hewlett et al., 2008). Perceived self-efficacy has been found to predict outcomes of a controlleddrinking programme in Australia (Sitharthan and
Kavanagh, 1990), and self-efficacy has been proven to be a powerful personal resource in coping with stress (Lazarus and Folkman, 1987). Chambliss and Murray (1979) published a US study showing that people who were overweight were most responsive to behavioural treatment when they had a high sense of self-efficacy. Self-efficacy in older women receiving an exercise intervention significantly predicted exercise behaviour at follow-up when controlling for biological and behavioural influences (McAuley, 1993). Increases in physical activity over time were associated with greater improvements in self-efficacy, which was in turn associated with improved physical function performance (McAuley et al., 2007).

Although there are no studies that focus on self-efficacy in communication or confidence in doctor-patient interactions in older people, many studies have shown that self-efficacy in communication is both measurable and modifiable. For example, patients' ratings of the effectiveness of professionals' communication are important predictors of the outcomes of diabetes self-management (Heisler et al., 2002).

The aim of the analysis reported in this paper is to measure self-efficacy in patient-doctor interactions in a sample of community-dwelling older people, and to explore factors associated with such self-efficacy, including the uptake of preventive care. Self-management may depend on the confidence an individual has in working with a doctor or a nurse as much as on their confidence in their own ability to initiate and sustain changes in their own behaviour. Self-efficacy in doctor-patient interactions is therefore a potentially important determinant of self-care for any given medical problem. Understanding factors associated with higher selfefficacy in doctor-patient interactions may be useful for clinicians to recognise and focus on those who have less confidence in their interactions with health-care professionals. In addition, it may be possible to measure the impact of interventions on modifying self-efficacy in doctorpatient interactions in older people.

\section{Hypotheses}

We hypothesised that lower self-efficacy in doctor-patient interactions would be associated with fewer years in education and lower income, higher consultation rates and lower uptake of 
preventive care. We hypothesised that there would be no significant associations with lifestyle (smoking, alcohol intake, high fat and low fibre intake), functional abilities and other demographic characteristics.

\section{Methods}

Three large group practices in suburban London were recruited to participate in a multi-centre, multinational randomised controlled trial investigating the effect of the Health Risk Appraisal for Older persons (HRA-O) on health behaviours and status (Stuck et al., 2002). Practices were purposively selected for their interest in primary care for older people, location in London (suburban) and routine use of electronic medical recording systems in clinical encounters. Local research ethics committee approval was obtained from Brent Medical Ethics Committee and King's College Hospital Research Ethics Committee. A full account of the methodology of the study is available elsewhere (Stuck et al., 2007), including recruitment of practices and patients, training of general practitioners in health promotion with older people, use of reminders and the evidence justifying the preventive care recommendations given. For more information on the HRA-O study and papers derived from it on the topics of social isolation, living alone, the experience of pain and predicting disablement, go to http:// www.ucl.ac.uk/pcph/dev/research-groups-themes/ age-stud-pub/previous-research/\#6 (UCL website for the HRA-O study). This paper is the fifth in a series aimed at a primary care audience.

To identify eligible patients aged 65 years and over, practice lists were cleaned by general practitioners. Eligibility criteria were: those living at home, without a) evidence of need for human assistance in basic activities of daily living, b) high dependency due to major physical or psychiatric illness, or cognitive impairment or c) a terminal illness. Patients also had to have a sufficient knowledge of English to complete the questionnaires. This patient population was further evaluated using the Probability of Recurrent Admissions (Pra) questionnaire (Pacala et al., 1995) and asked to complete a consent form by post. The Pra measures risk of hospital admission, and stratifies the population by level of risk for future in-patient care, and was used in the main study as the basis for risk-stratified outcome analyses.

Eligible and consenting patients were posted the HRA-O questionnaire. The HRA-O is a multidimensional, self-completion questionnaire that collects information on health, functional status, health behaviours, preventive care and psychosocial factors in older people (see Table 2). The development of the HRA-O questionnaire, the derivation of the instruments used in it, the exact definitions of categories (eg, 'low physical activity') and the feasibility of its use in British primary care have been reported elsewhere (Iliffe et al., 2005). Nonresponders to the initial mailing were sent a postal reminder. Those who responded, and those who did not report the need for human assistance in basic activities of daily living, were included in the study.

At the one-year follow-up, surviving participants were sent a HRA-O questionnaire with additional questions on health-care use. This follow-up questionnaire included all items required for outcome analysis. In addition, the one-year follow-up questionnaire was used for obtaining information on socio-economic information and self-reported chronic conditions among participants in the control and concurrent comparison groups. No reminders were sent to people not returning this questionnaire. To reduce the amount of missing information on preventative care, practices were asked to review patient medical records for information on preventative care use (vaccination coverage, blood glucose and cholesterol measurement, colon cancer screening) for patients who had returned the 1-year follow-up questionnaire but had incomplete information on some items of preventative care. In total, 97 patient records were used.

This questionnaire included five questions used to measure self-efficacy according to the PEPPI scale (see Box 1). 'Not confident' responses were scored as 1, 'confident' answers were scored as 3 and a score of 5 was given for a 'very confident' answer. The lowest possible score was 5 and the highest was 25 .

This was used to measure older patients' selfefficacy in obtaining medical information and attention to their medical concerns from doctors. Maly et al. (1998) concluded that PEPPI may be useful in measuring the impact of interventions designed to increase older patients' personal sense of effectiveness in obtaining needed health care. For example, it was used in a study in the 


\section{Box 1}

\section{Patient-Physician Interaction Score}

- How confident are you about the following...

- Knowing what questions to ask your doctor or nurse?

- Getting your doctor or nurse to answer all of your questions

- Making the most of your visit to the doctor or nurse

- Getting your doctor or nurse to take your main health concern seriously?

- Getting your doctor or nurse to do something about your main health concern

Netherlands, which showed that self-efficacy was significantly correlated with patients' satisfaction after physician encounters in primary care (Zandbelt et al., 2004). It was also used in a study showing that in low-income patients with prostate cancer, low self-efficacy for interacting with physicians was best predicted by diminished overall satisfaction with care, low confidence in providers and worse symptom distress (Maliski et al., 2004). Men with low self-efficacy fared worse over a range of psychosocial outcomes and both general and disease-specific health-related quality of life. It was also used to measure differences in self-efficacy between racial groups in a study looking at a public assistance programme for prostate cancer (Miller et al., 2008). This self-efficacy score was also used in a tailored education and coaching intervention to enhance care of cancer-related pain, as part of the Cancer Health Empowerment for Living without Pain study (Kravitz et al., 2009).

There appears to be very few other scales focussing on measuring self-efficacy in doctorpatient interactions. We used the five-point PEPPI scale because it was relatively brief and focussed on the doctor-patient interaction.

\section{Data analysis}

Data from the HRA-O questionnaires were entered on a database designed for the study, with double data entry for purposes of quality control, and analysed in a two-stage process using SPSS 12 for Windows. In the first stage of the analysis, $\chi^{2}$ tests were used to explore the associations between higher self-efficacy on the PEPPI scale and the characteristics listed in Table 2. Social isolation was measured according to the Lubben Social Network scale (Lubben, 1988; Lubben et al., 2006), and physical activity by the Physical Activity Scale for the Elderly score (Washburn et al., 1993). Other factors included were history of falls (Kelsey et al., 1992), assistance with more than one Lawton's Instrumental Activities of Daily Living (Lawton and Brody, 1969), self-reported functional change (Fried et al., 2000), more service utilisation (more than six visits to the doctor in the last 12 months) and polypharmacy (on four or more repeat medications). We included questions on how recently patients had had blood pressure measurements, cholesterol checks and random blood glucose tests to evaluate uptake of preventive care.

In the second stage of the analysis, the variables with a significant association (other than uptake of preventive care activities and dietary factors) were entered in a single forward step into a binary logistic regression model.

\section{Results}

In all, 5982 patients aged 65 years and over were identified across the three practices, of which 515 were excluded based on the exclusion criteria. In 2000, 5467 older people were sent an invitation letter, a consent form and the questionnaire. In 2002, 2989 participants were sent the HRA-O questionnaire containing the PEPPI scale, and $81 \%$ returned them. Of these, 2320 (78\%) completed all five PEPPI scale questions. Those who answered none or only some PEPPI questions were not significantly different from those who completed all five PEPPI questions in age or sex.

\section{Responses to the PEPPI questions}

Responses to each of the PEPPI questions are shown in Table 1.

\section{Analysis of PEPPI scores}

The mean total PEPPI score was 20 , with a median of 21 and a standard deviation of 5.383. The distribution of PEPPI scores was bimodal, peaking at 15 and 25 . One in three $(36 \%)$ of those who completed the PEPPI scale had scores of 15 or below, which for the purposes of analysis we 
Table 1 Responses to each of the PEPPI questions

\begin{tabular}{|c|c|c|c|}
\hline Self-reported confidence: & Very confident & Somewhat confident & Not confident \\
\hline Knowing what questions to ask your doctor or nurse & $1186 / 2384(50 \%)$ & $960 / 2384(40 \%)$ & $238 / 2384(10 \%)$ \\
\hline $\begin{array}{l}\text { Getting your doctor or nurse to answer all of your } \\
\text { questions }\end{array}$ & $1267 / 2376(53 \%)$ & $913 / 2376(38 \%)$ & 196/2376 (8\%) \\
\hline Making the most of your visit to the doctor or nurse & $1300 / 2377(55 \%)$ & $888 / 2377(37 \%)$ & 189/2377 (8\%) \\
\hline $\begin{array}{l}\text { Getting your doctor or nurse to take your main } \\
\text { health concern seriously }\end{array}$ & $1513 / 2377(64 \%)$ & $737 / 2377(31 \%)$ & $189 / 2377(5 \%)$ \\
\hline $\begin{array}{l}\text { Getting your doctor or nurse to do something } \\
\text { about your main health concern }\end{array}$ & $1524 / 2376(64 \%)$ & $720 / 2376(30 \%)$ & $132 / 2376(6 \%)$ \\
\hline
\end{tabular}

Table 2 Factors associated with higher self-efficacy: bivariate analysis $n=1485$

\begin{tabular}{|c|c|c|c|c|c|}
\hline Characteristic & $n$ & $\%$ & $\begin{array}{l}\text { Odds } \\
\text { ratio }\end{array}$ & $\begin{array}{l}95 \% \text { confidence } \\
\text { intervals }\end{array}$ & $P$-value \\
\hline \multicolumn{6}{|l|}{ Demographics } \\
\hline Female & 797 & 51.7 & 0.79 & $0.66-0.94$ & 0.007 \\
\hline Age over 75 & 642 & 41.6 & 1.01 & $0.85-1.20$ & 0.91 \\
\hline Basic education only & 922 & 60.4 & 0.77 & $0.66-0.93$ & 0.006 \\
\hline State pension only & 489 & 32.1 & 1.14 & $0.95-1.37$ & 0.17 \\
\hline Lives alone & 463 & 30.3 & 0.80 & $0.66-0.96$ & 0.015 \\
\hline \multicolumn{6}{|l|}{ BMI } \\
\hline $\mathrm{BMI}<20$ underweight & 57 & 3.9 & 1.02 & $0.64-1.62$ & 0.94 \\
\hline BMI >27 overweight/obese & 495 & 34.1 & 0.87 & $0.72-1.05$ & 0.14 \\
\hline \multicolumn{6}{|l|}{ Health services use } \\
\hline More doctor visits & 1279 & 83.6 & 1.12 & $0.89-1.41$ & 0.32 \\
\hline Four or more repeat medicines & 495 & 34.7 & 1.05 & $0.87-1.26$ & 0.63 \\
\hline \multicolumn{6}{|l|}{ Psychological well-being } \\
\hline Depressed mood & 200 & 13.1 & 0.45 & $0.37-0.57$ & $<0.001$ \\
\hline Memory problems & 106 & 7.1 & 0.40 & $0.31-0.53$ & $<0.001$ \\
\hline At risk of social isolation & 182 & 12.0 & 0.57 & $0.45-0.72$ & $<0.001$ \\
\hline \multicolumn{6}{|l|}{ Functional ability, activity and falls } \\
\hline Difficulty with or need for assistance in one or more IADLs & 539 & 36.0 & 0.72 & $0.60-0.86$ & $<0.001$ \\
\hline Decreased activity frequency & 536 & 36.5 & 0.73 & $0.61-0.87$ & 0.01 \\
\hline Multiple falls in last year & 163 & 10.9 & 0.84 & $0.64-1.10$ & 0.20 \\
\hline Limited activities due to fear of falling & 354 & 23.3 & 0.62 & $0.51-0.75$ & $<0.001$ \\
\hline \multicolumn{6}{|l|}{ Lifestyle } \\
\hline Current tobacco use & 136 & 9.4 & 0.82 & $0.61-1.10$ & 0.20 \\
\hline Hazardous alcohol use & 308 & 20.6 & 1.05 & $0.84-1.31$ & 0.67 \\
\hline High fat consumption & 1279 & 88.1 & 0.96 & $0.73-1.26$ & 0.74 \\
\hline Low fibre/fruit consumption & 917 & 62.1 & 0.75 & $0.62-0.90$ & 0.002 \\
\hline Low level of physical activity & 338 & 23.0 & 0.83 & $0.68-1.02$ & 0.08 \\
\hline \multicolumn{6}{|l|}{ Preventive care checks } \\
\hline Fasting blood glucose in the past 3 years & 434 & 28.1 & 1.62 & $1.32-2.01$ & 0.01 \\
\hline Blood pressure check in the last year & 1324 & 85.9 & 1.61 & $1.29-2.02$ & $<0.001$ \\
\hline Cholesterol check in past 5 years & 860 & 55.8 & 1.43 & $1.20-1.70$ & $<0.001$ \\
\hline Influenza vaccination in the past year & 1282 & 83.1 & 1.31 & $1.05-1.63$ & 0.016 \\
\hline Pneumonia vaccine in the past & 521 & 33.8 & 1.18 & $0.98-1.43$ & 0.08 \\
\hline
\end{tabular}

$\mathrm{BMI}=$ body mass index; $\mathrm{IADL}=$ instrumental activities of daily living.

categorised as 'lower self-efficacy'. Scores of 16 or more were categorised as 'higher self-efficacy'. There were 835 people in the lower self-efficacy category and 1485 in the higher self-efficacy category.
First stage analysis

Table 2 shows the associations between the chosen HRA-O domains and higher self-efficacy as measured by the PEPPI scale. Higher self-efficacy

Primary Health Care Research \& Development 2011; 12: 348-356 
Table 3 Correlation of lower self-efficacy with selected factors significantly associated in bivariate analyses: results of multivariate logistic regression analysis

\begin{tabular}{llll}
\hline & Odds ratio & 95\% confidence intervals & $P$-value \\
\hline Age $<75$ & 1.22 & $0.99-1.51$ & 0.06 \\
Female & 0.89 & $0.73-1.10$ & 0.28 \\
Basic education only & 0.90 & $0.73-1.12$ & 0.35 \\
Self-rated memory problems & 0.49 & $0.35-0.67$ & $<0.001$ \\
At risk of social isolation & 0.63 & $0.48-0.82$ & $<0.001$ \\
Low mood & 0.60 & $0.47-0.78$ & 0.001 \\
Difficulty with or need for assistance in one or more IADLs & 1.05 & $0.83-1.32$ & 0.70 \\
Decreased activity frequency & 0.94 & $0.75-1.17$ & 0.56 \\
Limited activities due to fear of falling & 0.71 & $0.55-0.90$ & 0.001 \\
\hline
\end{tabular}

$\mathrm{IADL}=$ instrumental activities of daily living.

was significantly less likely among women, those with only basic education, and those living alone. Service utilisation (frequent doctor visits), polypharmacy and most lifestyle characteristics (smoking, hazardous alcohol consumption, dietary habits) were not significantly associated with higher or lower self-efficacy. However, those who reported having an unhealthy diet with low fruit and fibre content were less likely to have higher self-efficacy. Those reporting impaired functional ability, fear of falling and low physical activity levels were also less likely to have high self-efficacy scores. Multiple falls in the previous 12 months were unrelated to self-efficacy levels. Participants in this study were significantly less likely to have a higher self-efficacy in doctor-patient interactions if they had depressed mood, reported memory problems or were at risk of social isolation.

\section{Second stage analysis}

The likelihood of having higher self-efficacy in doctor-patient interactions remained significantly negatively associated with memory problems, low mood, risk of social isolation and limitation of activities due to fear of falling, after adjustment for age, gender and years in education (see Table 3 ). Functional losses were no longer significantly associated with self-efficacy.

Those with higher self-efficacy were significantly more likely to have had recent measurements of BP, cholesterol and fasting blood glucose, and influenza immunisation.

\section{Discussion}

To the best of our knowledge, this is the first study of self-efficacy in doctor-patient interaction in older people in British general practice. Two-thirds of this sample of older people had PEPPI scores suggesting higher self-efficacy, and they were significantly more likely to have had recent measurements of blood pressure, cholesterol and fasting blood glucose, and influenza immunisation. A third had lower self-efficacy, and there were significant associations between having low self-efficacy and having poor selfrated memory, only basic education, limitation of activities due to fear of falling, being at risk of social isolation and having depressed mood.

Our hypothesis that lower self-efficacy would be associated with fewer years in education and lower uptake of preventive care was supported by the findings of the bivariate analyses, but not in the logistic regression analysis. The expected association of lower self-efficacy with lower income was not supported. The prediction that self-efficacy in doctor-patient interactions would be independent of other demographic and functional characteristics was supported by the findings from the logistic regression analysis. The relationship between depression and selfefficacy needs further exploration in longitudinal studies, to allow causality to be determined, for it is possible that low confidence is the product of depression, not a cause. The possibility that self-efficacy mediates between impairments or disability and depression also needs further investigation. The extent to which self-efficacy in doctor-patient interactions is modifiable needs to be evaluated, and this may require improved methods for measuring self-efficacy in primary care. 


\section{Limitations of the study}

Owing to the cross-sectional nature of the data, it is not possible to determine causality in the relationships between self-efficacy and the factors associated with it. The sample was drawn from three general practices in suburban London, and subject to eligibility criteria and disability screening implemented for recruitment into a trial of health promotion, which may limit the generalisability of the results. The distribution of self-efficacy scores found in this sample may be different from that in the general population of older primary care patients, partly because we deliberately excluded disabled older people, people needing assistance with basic activities of daily living, and people with mental health problems and dementia, and partly because the participants were a self-selecting sub-group who returned lengthy questionnaires. We cannot comment on those who did not choose to participate in the questionnaire study. The results showed a bimodal distribution of results, which again cannot be explained, but may need further study to evaluate its significance. Finally, self-report of diagnoses and of preventive care uptake may be inaccurate.

\section{Comparison with existing literature}

There are no published studies with regard to self-efficacy in doctor-patient interactions in the older people. The association between depressed mood and low self-efficacy has been noted in other studies, although is not a dominant theme in the discussion of self-efficacy as a modifiable determinant of health. For example, in a study of adherence to antihypertensive medication in African Americans, depressive symptoms were associated with poor medication adherence and low self-efficacy (Schoenthaler et al., 2007) but this relationship became non-significant when controlling for self-efficacy. The authors saw self-efficacy as having a mediating role between depression and medication adherence. In US studies, a similar mediating role has also been found between limited social support and depression (Holahan and Holahan, 1987), and between pain intensity and disability (Arnstein et al., 1999).

Another way of thinking about self-efficacy is shown by a study of patients with heart failure in which patients were characterised as 'experts', 'novices' and 'inconsistent' in a recent self-care management study in the United States (Dickson et al., 2008). 'Experts' had experience and skill in self-care, which novices lacked, and positive attitudes with self-efficacy that aligned with their behaviours. Most patients $(71 \%)$ were classified as 'inconsistent', a self-care type associated with impaired cognition, poor physical functioning, negative attitudes and poor self-efficacy. Our findings are strikingly similar in the associations between lower self-efficacy and memory problems, low mood and fear of falling.

\section{Implications for clinical practice and future research}

Improving self-efficacy in any aspect of health is no panacea. The concept and much of the clinical practice based on fostering self-efficacy has been part of health care for twenty years, and (with some exceptions such as diabetes, arthritis and asthma management) have had limited impact on health behaviours. For example, in a recent Canadian study designed to increase selfefficacy in balance and mobility, both resistance training and agility training significantly improved balance confidence by a modest $6 \%$ from baseline after 13 weeks (Liu-Ambrose et al., 2004). The change in balance confidence was only weakly correlated with improved general physical function and not significantly correlated with the changes in fall risk score, postural stability, gait speed or physical activity level. The authors also observed enhanced confidence in balance in people whose falls risk increased or physical abilities deteriorated. Our paper identifies characteristics that suggest that an older person will be less likely to have confidence in their interactions with doctors and their health, so that theoretically this subgroup of patients could benefit from interventions to improve their self-efficacy. However, even with approaches designed to enhance self-efficacy, changing behaviour through professional intervention remains difficult (Borsody et al., 1999).

\section{Conclusion}

A third of older people in this study had low selfefficacy in doctor-patient interactions, and they appeared to be a vulnerable group. The population prevalence of lower self-efficacy in doctor-patient interactions may be larger. Low mood and poor 
memory are both symptoms of depression, and limitation of activity because of the fear of falling has a psychological component independent of the experience of falling. In this population of older people, low self-efficacy in interactions with doctors (as measured by the PEPPI scale) may be a symptom of depression, or a characteristic of older people who also experience social isolation and depression. Understanding a person's confidence in doctor-patient interactions as measured by self-efficacy may be useful in clinical practice, but policies that depend on enhancing self-care and self-management need to consider the large number of older people with low selfefficacy in using medical services. Further studies looking at whether self-efficacy in doctor-patient interactions is modifiable and improvable may be useful.

\section{Acknowledgements}

Local research ethics committee approval was obtained from Brent Medical Ethics Committee (BEC745) and King's College Hospital Research Ethics Committee (Protocol 01-010). This study was funded by the European Commission project QLK6-CT-1999-02205 and the Federal Education Science Ministry, Berne, Switzerland BBW 990311.1. The authors have no conflict of interest to declare. The authors thank the practices and patients involved in the study.

\section{References}

Arnstein, P., Caudill, M., Mandle, C., Norris, A. and Beasley, R. 1999: Self-efficacy as a mediator of the relationship between pain intensity, disability and depression in chronic pain patients. Pain 80, 483-91.

Bandura, A. 1977: Self-efficacy: toward a unifying theory of behavior change. Psychological Review 84, 191-215.

Bandura, A. 2004: Health promotion by social cognitive means. Health Education and Behavior 31, 143-64.

Bodenheimer, T., Lorig, K., Holman, H. and Grumbach, K. 2002: Patient self-management of chronic disease in primary care. Journal of the American Medical Association 288, 2469-475.

Borsody, J., Courtney, M., Taylor, K. and Jaraith, N. 1999: Using self-efficacy to increase physical activity in patients with heart failure. Home Healthcare Nurse 17, 113-18.

Chambliss, C. and Murray, E. 1979: Efficacy attribution, locus and weight loss. Cognitive Therapy and Research 3, 349-53.
Dickson, V.V., Deatrick, J.A. and Riegel, B. 2008: A typology of heart failure self-care management in non-elders. European Journal of Cardiovasular Nursing 7, 171-81.

Fried, L.P., Bandeen-Roche, K., Chaves, P.H.M. and Johnson, B.A. 2000: Pre-clinical mobility predicts incident mobility disability in older women. Journal of Gerontology 55A, M43-52.

Gerin, W., Litt, M., Deich, J. and Pickering, T. 1995: Selfefficacy as a moderator of perceived control effects on cardiovascular reactivity: is enhanced control always beneficial? Psychosomatic Medicine 57, 390-97.

Heisler, M., Bouknight, R., Hayward, R., Smith, D. and Kerr, E. 2002: The relative importance of physician communication, participatory decision making, and patient understanding in diabetes self-management. Journal of General Internal Medicine 17, 243-52.

Hewlett, S., Cockshott, Z., Almeida, C., Richards, P., Lowe, R., Greenwood, R., Kirwan, J. and for the RASE Study Group. 2008: Sensitivity to change of the Rheumatoid Arthritis Self-Efficacy scale (RASE) and predictors of change in self-efficacy. Musculoskeletal Care 6, 49-67.

Holahan, C.K. and Holahan, C.J. 1987: Self-efficacy, social support and depression in aging: a longitudinal analysis. Journal of Gerontology 42, 65-68.

Iliffe, S., Kharicha, K., Harari, D., Swift, C. and Stuck, A. 2005: Health risk appraisal for older people in general practice using an expert system: a pilot study. Health and Social Care in the Community 13, 21-29.

Kelsey, J.L., Browner, W.S., Seeley, D.G., Nevitt, M.C. and Cummings, S.R. 1992: Risk factors for fractures of the distal forearm and proximal humerus. American Journal of Epidemiology 135, 477-89.

Kravitz, R.L., Tancredi, D.J., Street, R.L. Jr, Kalauokalani, D., Grennan, T., Wun, T., Slee, C., Evans Dean, D., Lewis, L., Saito, N. and Franks, P. 2009: Cancer Health Empowerment for Living without Pain (Ca-HELP): study design and rationale for a tailored education and coaching intervention to enhance care of cancer-related pain. BMC Cancer 9, 319.

Lawton, M.P. and Brody, E.M. 1969: Assessment of older people: self maintaining and instrumental activities of daily living. Gerontologist 9, 179-86.

Lazarus, R.S. and Folkman, S. 1987: Transactional theory and research and coping. European Journal of Personality 1, 141-70.

Liu-Ambrose, T., Khan, K.M., Eng, J.J., Lord, S.R. and McKay, H.A. 2004: Balance confidence improves with resistance or agility training. Increase is not correlated with objective changes in fall risk and physical abilities. Gerontology 50, 373-82.

Lorig, K., Stewart, A., Ritter, P., Gonzalez, V., Laurent, D. and Lynch, J. 1996: Outcome measures for health education and other health care interventions. California: Sage, 5pp.

Lubben, J.E. 1988: Assessing social networks among elderly populations. Family and Community Health 11, 42-52.

Lubben, J., Blozik, E., Gillmann, G., Iliffe, S., von RentelnKruse, W., Beck, J.C. and Stuck, A.E. 2006: Performance of an abbreviated version of the Lubben social network scale 
among three European community-dwelling older adult populations. Gerontologist 46, 503-13.

Maliski, S.L., Kwan, L., Krupski, T., Fink, A., Orecklin, J.R. and Litwin, M.S. 2004: Confidence in the ability to communicate with physicians among low-income patients with prostate cancer. Urology 64, 329-34.

Maly, R.C., Frank, J.C., Marshall, G.N., DiMatteo, M.R. and Reuben, D.B. 1998: Perceived efficacy in patient physician interactions (PEPPI): validation of an instrument in older persons. Journal of the American Geriatrics Society 46, 889-94.

McAuley, E. 1993: Self-efficacy and the maintenance of exercise participation in older adults. Journal of Behavioral Medicine 16, 103-13.

McAuley, E., Morris, K.S., Doerksen, S.E., Motl, R.W., Liang, H., White, S.M., Wójcicki, T.R. and Rosengren, K. 2007: Effects of change in physical activity on physical function limitations in older women: mediating roles of physical function performance and self-efficacy. Journal of the American Geriatrics Society 55, 1967-973.

Miller, D.C., Gelberg, L., Kwan, L., Stepanian, S., Fink, A., Andersen, R.M. and Litwin, M.S. 2008: Racial disparities in access to care for men in a public assistance program for prostate cancer. Journal of Community Health 33, 318-35.

Ogedegbe, G., Mancuso, C.A., Allegrante, J.P. and Charlson, M.E. 2003: Development and evaluation of a medication adherence self-efficacy scale in hypertensive African-American patients. Journal of Clinical Epidemiology 56, 520-29.

Pacala, J.T., Boult, C. and Boult, L. 1995: Predictive validity of a questionnaire that identifies older persons at risk for hospital admission. Journal of the American Geriatrics Society 43(4), 374-77.

Resnick, B. and Jenkins, L. 2000: Testing the reliability and validity of the self-efficacy for exercise scale. Nursing Research 49, 154-59.
Schoenthaler, A., Ogedegbe, G. and Allegrante, J.P. 2007: Self-efficacy mediates the relationship between depressive symptoms and medication adherence among hypertensive African Americans. Health Education and Behavior 36, 127-37.

Sitharthan, T. and Kavanagh, D.J. 1990: Role of self-efficacy in predicting outcomes from a programme for controlled drinking. Drug and Alcohol Dependence 27, 87-94.

Strecher, V., DeVellis, B. and Rosenstock, I. 1986: The role of self-efficacy in achieving health behavior change. Health Education and Behavior 13, 73-92.

Stuck, A.E., Elkuch, P., Dapp, U., Anders, J., Iliffe, S. Swift, C. and for the Pro-Age pilot study group. 2002: Feasibility and yield of a self-administered questionnaire for health risk appraisal in older people in three European countries. Age and Ageing 31, 463-67.

Stuck, A.E., Kharicha, K., Dapp, U., Anders, J., von RentelnKruse, W., Meier-Baumgartner, H., Iliffe, S., Harari, D., Bachmann, M.D., Egger, M., Gilman, G., Beck, J. and Swift, C. 2007: The ProAge study: an international randomised controlled study of health risk appraisal for older persons based in general practice. BMC Medical Research Methodology 7, 2.

UCL website for the HRA-O study. Retrieved 24 February 2011 from http://www.ucl.ac.uk/pcph/dev/research-groupsthemes/age-stud-pub/previous-research/\#6

Washburn, R.A., Smith, K.W., Jette, A.M. and Janney, T. 1993: The physical activity scale for the elderly (PASE): development and evaluation. Journal of Clinical Epidemiology 46, 153-62.

Zandbelt, L., Smets, E., Oort, F., Godfried, M. and De Haes, H.C. 2004: Satisfaction with the outpatient encounter a comparison of patients' and physicians'. Views Journal of General Internal Medicine 19, 1088-095. 